HU-EP-17/06

\title{
Strings on the lattice and AdS/CFT
}

\author{
Valentina Forini* \\ Humboldt-Universität zu Berlin, Zum Großen Windkanal 6, 12489 Berlin, Germany \\ E-mail: foriniephysik.hu-berlin.de
}

\section{Lorenzo Bianchi}

II. Institut für Theoretische Physik, Universität Hamburg, Luruper Chaussee 149, 22761

Hamburg, Germany

E-mail: Iorenzo.bianchi@desy.de

\section{Bjoern Leder}

Institutsrechenzentrum, Institut für Physik

Humboldt-Universität zu Berlin, Newtonstr. 15, 12489 Berlin, German

E-mail: leder@physik.hu-berlin. de

\section{Philipp Töpfer}

Humboldt-Universität zu Berlin, Zum Großen Windkanal 6, 12489 Berlin, Germany

E-mail: philipp.toepfer@physik.hu-berlin.de

\section{Edoardo Vescovi}

Humboldt-Universität zu Berlin, Zum Großen Windkanal 6, 12489 Berlin, Germany Institute of Physics, University of São Paulo, Rua do Matão 1371, 05508-090 São Paulo, Brazil

E-mail: vescovieif.usp.br

We present a new auxiliary field representation for the four-fermi term of the gauge-fixed Green-Schwarz superstring action which describes fluctuations around the null-cusp background in $A d S_{5} \times S^{5}$. We sketch the main features of the fermionic spectrum, identifying the region of parameter space where the sign ambiguity is absent. Measurements for the observables in the setup here described are presented and discussed in [1].

34th annual International Symposium on Lattice Field Theory

24-30 July 2016

University of Southampton, United Kingdom

\footnotetext{
*Speaker.
} 


\section{Discussion}

Investigating via lattice field theory methods quantum string worldsheets relevant in AdS/CFT has recently become a concrete possibility $[2-4]^{1}$. The model under study is the AdS-lightcone gauge-fixed (Type IIB Green-Schwarz) superstring action [6] describing fluctuations around the classical string solution ending, at the AdS boundary, on a lightlike cusped Wilson loop [7]. It consists of a highly non-trivial two-dimensional quantum field theory with quartic fermionic interactions; its perturbative analysis provides, according to AdS/CFT, strong coupling information on the behaviour of several important observables in the dual gauge theory and has been explicitly performed up to two loops in sigma-model semiclassical quantization [7].

In [2-4] lattice simulations employing a Rational Hybrid Monte Carlo (RHMC) algorithm were performed in order to measure the vacuum expectation value of the action - from which the cusp anomaly of $\mathscr{N}=4$ super Yang-Mills [2-4] can be extracted - and the mass [3,4] of the two AdS excitations transverse to the relevant null cusp classical string solution. For both these observables it is possible to compare the results with the behavior predicted via integrability [8] at various values of the coupling $g=\sqrt{\lambda} /(4 \pi)$. At large $g$, i.e. the perturbative regime of the sigma-model, for both the observables a good agreement was found. At lower values of $g$, after a non-perturbative subtraction of quadratic divergences, a complex phase in the fermion determinant - in fact, a Pfaffian - was detected [3,4]. There, it was also concluded that a strong sign problem in simulations would appear for values of the (lattice) coupling $g \leq 5$.

The origin of such complex phase is a non-hermitian piece in the Lagrangian, a specific Yukawa-like term resulting from the standard linearization of a quartic fermionic interaction which appears originally as a "repulsive" potential [4]. In this contribution we present a new auxiliary field representation of the four-fermi term, following an algebraic manipulation of the original fermionic Lagrangian inspired by [9] (see also [10]). The result is a Lagrangian linear in fermions which is fully hermitian, and a quadratic fermionic operator $O_{F}$ which - in presence or not of Wilson-like terms - is antisymmetric and obeys a constraint reminiscent of the " $\gamma_{5}$-hermiticity" in lattice QCD. These two properties ensure that $\operatorname{det} O_{F}$ is real and non-negative, from which a real Pfaffian $\left(\operatorname{Pf} O_{F}\right)^{2}=\operatorname{det} O_{F} \geq 0$. Eliminating the complex phase allows us to eliminate a systematic error in measurements ${ }^{2}$. As a sign ambiguity remain in its definition, $\operatorname{Pf} O_{F}= \pm \sqrt{\operatorname{det} O_{F}}$, the non definite positive Pfaffian can not be directly simulated using a (rational) hybrid Monte Carlo algorithm, but only upon replacement $\operatorname{Pf} O_{F} \rightarrow \operatorname{det}\left(O_{F}^{\dagger} O_{F}\right)^{\frac{1}{4}}$ and a reweighting procedure which can potentially break down if the sign ambiguity is severe. Below, after presenting the details of the new linearization, we show numerically some relevant features of the spectrum of the new quadratic fermionic operator. The origin of the sign ambiguity appears to be related to the Yukawa-like terms, this time including those present in the original Lagrangian (before linearization). Also, one may identify the region of parameter space where no sign flips can occur, and that therefore cannot be affected by a sign problem. As the latter occurs at $g \sim 2$ and lower, this leaves the possibility of "safe" measurements at e.g. $g=10$, where features of the non-perturbative regime appear to be detected by (previous [4] and) current [1] simulations. The measurements of relevant observables in this novel setup are discussed in the forthcoming [1].

\section{Linearization}

The euclidean superstring action in AdS-lightcone gauge-fixing [6] describing quantum fluc-

\footnotetext{
${ }^{1}$ For lattice investigations in other models relevant in AdS/CFT see e.g. [5].

${ }^{2}$ In order to treat it via standard reweighting, the phase should be calculated explicitly. As it is highly non trivial to evaluate efficiently complex determinants for arbitrarily big matrices, in [4] this was done only for small lattices, i.e. small values of lattice points $N$. Observing that the reweighting had no effect on the central value of the observables under study, the phase was omitted from the simulations in order to consistently take the continuum limit $(N \rightarrow \infty)$, and in absence of data for larger lattices the possible systematic error related to this procedure was not assessed.
} 
tuations around the null-cusp background in $A d S_{5} \times S^{5}$ reads [7]

$S_{\text {cusp }}=g \int d t d s\left\{\left|\partial_{t} x+\frac{1}{2} x\right|^{2}+\frac{1}{z^{4}}\left|\partial_{s} x-\frac{1}{2} x\right|^{2}+\left(\partial_{t} z^{M}+\frac{1}{2} z^{M}+\frac{i}{z^{2}} z_{N} \eta_{i}\left(\rho^{M N}\right)^{i}{ }_{j} \eta^{j}\right)^{2}\right.$
$+\frac{1}{z^{4}}\left(\partial_{s} z^{M}-\frac{1}{2} z^{M}\right)^{2}+i\left(\theta^{i} \partial_{t} \theta_{i}+\eta^{i} \partial_{t} \eta_{i}+\theta_{i} \partial_{t} \theta^{i}+\eta_{i} \partial_{t} \eta^{i}\right)-\frac{1}{z^{2}}\left(\eta^{i} \eta_{i}\right)^{2}$
$\left.+2 i\left[\frac{1}{z^{3}} z^{M} \eta^{i}\left(\rho^{M}\right)_{i j}\left(\partial_{s} \theta^{j}-\frac{1}{2} \theta^{j}-\frac{i}{z} \eta^{j}\left(\partial_{s} x-\frac{1}{2} x\right)\right)+\frac{1}{z^{3}} z^{M} \eta_{i}\left(\rho_{M}^{\dagger}\right)^{i j}\left(\partial_{s} \theta_{j}-\frac{1}{2} \theta_{j}+\frac{i}{z} \eta_{j}\left(\partial_{s} x-\frac{1}{2} x\right)^{*}\right)\right]\right\}$

where $x, x^{*}$ are two bosonic fields transverse to the subspace $A d S_{3}$ of the classical solution and $z^{M}(M=1, \cdots, 6)$ are the bosonic fileds of the $A d S_{5} \times S^{5}$ spacetime in Poincare patch, with $z=$ $\sqrt{z_{M} z^{M}}$. The Graßmann-odd fields $\theta_{i}, \eta_{i}, i=1,2,3,4$ are complex variables (no Lorentz spinor indices appear), such that $\theta^{i}=\left(\theta_{i}\right)^{\dagger}, \eta^{i}=\left(\eta_{i}\right)^{\dagger}$ and transforming in the fundamental representation of the $S U(4)$ R-symmetry group. The matrices $\rho_{i j}^{M}$ are the off-diagonal blocks of $S O(6)$ Dirac matrices $\gamma^{M}$ in chiral representation, and $\left(\rho^{M N}\right)_{i}^{j}=\left(\rho^{[M} \rho^{\dagger N]}\right)_{i}^{j}$ are the $S O(6)$ generators. In the action (2.1) a massive parameter $\left(\sim P_{+}\right)$is missing, which we restore below in (2.11) defining it as $m$.

As standard, to take into account the fermionic contribution in the case of higher-order interactions one linearizes the corresponding Lagrangian and then formally integrates out the Graßmannodd fields letting their determinant - here, a Pfaffian - to enter the Boltzmann weight of each configuration through re-exponentiation

$$
\int D \Psi e^{-\int d t d s \Psi^{T} O_{F} \Psi}=\operatorname{Pf} O_{F} \longrightarrow\left(\operatorname{det} O_{F} O_{F}^{\dagger}\right)^{\frac{1}{4}}=\int D \xi D \bar{\xi} e^{-\int d t d s \bar{\xi}\left(O_{F} O_{F}^{\dagger}\right)^{-\frac{1}{4}} \xi}
$$

Here, we focus on the part of the Lagrangian in (2.1) which is quartic in fermion

$$
\mathscr{L}_{4}=\frac{1}{z^{2}}\left[-\left(\eta^{2}\right)^{2}+\left(i \eta_{i}\left(\rho^{M N}\right)_{j}^{i} n^{N} \eta^{j}\right)^{2}\right]
$$

where $n^{M}=\frac{z^{M}}{z}$. Notice the plus sign in front of the second term in (2.3), which squares an hermitian bilinear $\left(i \eta_{i} \rho^{M N^{i}}{ }_{j} \eta^{j}\right)^{\dagger}=i \eta_{j} \rho^{M N}{ }_{i}{ }_{i}^{i}[4]$. Then the standard Hubbard-Stratonovich transformation

$$
\begin{aligned}
& \exp \left\{-g \int d t d s\left[-\frac{1}{z^{2}}\left(\eta^{i} \eta_{i}\right)^{2}+\left(\frac{i}{z^{2}} z_{N} \eta_{i} \rho^{M N^{i}}{ }_{j} \eta^{j}\right)^{2}\right]\right\} \\
& \quad \sim \int D \phi D \phi^{M} \exp \left\{-g \int d t d s\left[\frac{1}{2} \phi^{2}+\frac{\sqrt{2}}{z} \phi \eta^{2}+\frac{1}{2}\left(\phi_{M}\right)^{2}-i \frac{\sqrt{2}}{z^{2}} \phi^{M} z_{N}\left(i \eta_{i} \rho^{M N^{i}}{ }_{j} \eta^{j}\right)\right]\right\}
\end{aligned}
$$

generates a non-hermitian term, the last one above, resulting in a complex-valued Pfaffian for the fermionic operator. Here we provide a solution to this problem by rewriting the Lagrangian (2.3) in a suitable form. The procedure is inspired by [9], where a simpler action with $\mathrm{SO}(4)$ fourfermion terms in three dimensions was considered (see also the four-dimensional $S U(4)$ counterpart in [10]). The Lagrangian (2.3) is invariant under $S U(4) \times U(1)$ transformations and this requires a generalization of [9]. Let us start by eliminating the matrices $\rho^{M N}$ from the second term of (2.3) in favour of $\rho^{M}$. After some $\rho$-matrices manipulations we get

$$
\mathscr{L}_{4}=\frac{1}{z^{2}}\left(-4\left(\eta^{2}\right)^{2}+2\left|\eta_{i}\left(\rho^{N}\right)^{i k} n_{N} \eta_{k}\right|^{2}\right)
$$

where the plus sign in front of the second term still prevents a real Pfaffian after the HubbardStratonovich transformation. We then define a duality transformation, reminiscent of the standard 
Hodge duality, but adapted to our particular case. Given $\Sigma_{i}{ }^{j} \equiv \eta_{i} \eta^{j}$ the dual matrix $\tilde{\Sigma}_{j}{ }^{i}$ is defined by

$$
\tilde{\Sigma}_{j}^{i}=n_{N} n_{L}\left(\rho^{N}\right)^{i k}\left(\rho^{L}\right)_{j l} \Sigma_{k}{ }^{l}
$$

Notice that $\tilde{\tilde{\Sigma}}=\Sigma$ and $\Sigma^{i}{ }_{j} \equiv\left(\Sigma_{i}{ }^{j}\right)^{\dagger}=\Sigma_{j}{ }^{i}$. One can then easily rewrite the quartic Lagrangian as

$$
\mathscr{L}_{4}=\frac{2}{z^{2}} \operatorname{Tr}(\Sigma \Sigma+\tilde{\Sigma} \tilde{\Sigma}-\Sigma \tilde{\Sigma})
$$

where the trace is over $S U(4)$ fundamental indices. Although we split the first two terms in (2.7) to exhibit the neutrality of the Lagrangian under duality transformation it is useful to keep in mind that $\operatorname{Tr} \tilde{\Sigma} \tilde{\Sigma}=\operatorname{Tr} \Sigma \Sigma$. Since we want to write down a Lagrangian as the sum of two terms squared, it is natural to introduce the self- and antiself-dual part of $\Sigma$

$$
\Sigma_{ \pm}=\Sigma \pm \tilde{\Sigma}
$$

such that $\tilde{\Sigma}_{ \pm}= \pm \Sigma_{ \pm}$. Now the crucial, though elementary fact that $\operatorname{Tr} \Sigma_{ \pm} \Sigma_{ \pm}=2 \operatorname{Tr}(\Sigma \Sigma \pm \Sigma \tilde{\Sigma})$ gives us some freedom in the choice of the sign in the Lagrangian since

$$
\mathscr{L}_{4}=\frac{1}{z^{2}} \operatorname{Tr}\left(4 \Sigma \Sigma \mp \Sigma_{ \pm} \Sigma_{ \pm} \pm 2 \Sigma \Sigma\right) .
$$

This last equation proves that the sign problem was an artefact of our naive linearization. Indeed (2.9) provides two equivalent forms of the same action, one which would lead to a sign problem and one which would not. Choosing the latter, i.e. the one involving $\Sigma_{+}$, we get

$$
\mathscr{L}_{4}=\frac{1}{z^{2}}\left(-6\left(\eta^{2}\right)^{2}-\Sigma_{+}^{j}{ }_{i}^{j} \Sigma_{+j}^{i}\right)
$$

In this form the Lagrangian is suitable for a Hubbard-Stratonovich transformation. In particular we have

$$
\begin{aligned}
\exp \left\{-g \int d t d s\left[-\frac{1}{z^{2}}(\right.\right. & \left.\left.\left.-6\left(\eta^{2}\right)^{2}-\Sigma_{+i}^{j} \Sigma_{+j}^{i}\right)\right]\right\} \\
& \sim \int D \phi D \phi^{M} \exp \left\{-g \int d t d s\left[\frac{12}{z} \eta^{2} \phi+6 \phi^{2}+\frac{2}{z} \Sigma_{+}^{i} \phi_{i}^{j}+\phi_{j}^{i} \phi_{i}^{j}\right]\right\}
\end{aligned}
$$

where $\phi_{j}^{i}$ can be thought of as a $4 \times 4$ complex hermitian matrix with 16 real degrees of freedom. Therefore the new linearization proposed here introduces a total of 17 auxiliary fields.

The final form of the Lagrangian is then

$$
\begin{aligned}
\mathscr{L} & =\left|\partial_{t} x+\frac{m}{2} x\right|^{2}+\frac{1}{z^{4}}\left|\partial_{s} x-\frac{m}{2} x\right|^{2}+\left(\partial_{t} z^{M}+\frac{m}{2} z^{M}\right)^{2}+\frac{1}{z^{4}}\left(\partial_{s} z^{M}-\frac{m}{2} z^{M}\right)^{2} \\
& +6 \phi^{2}+\phi_{j}^{i} \phi_{i}^{j}+\psi^{T} O_{F} \psi
\end{aligned}
$$

with $\psi \equiv\left(\theta^{i}, \theta_{i}, \eta^{i}, \eta_{i}\right)$ and

$$
O_{F}=\left(\begin{array}{cccc}
0 & \mathrm{i} \partial_{t} & -\mathrm{i} \rho^{M}\left(\partial_{s}+\frac{m}{2}\right) \frac{z^{M}}{z^{3}} & 0 \\
\mathrm{i} \partial_{t} & 0 & 0 & -\mathrm{i} \rho_{M}^{\dagger}\left(\partial_{s}+\frac{m}{2}\right) \frac{z^{M}}{z^{3}} \\
\mathrm{i} \frac{z^{M}}{z^{3}} \rho^{M}\left(\partial_{s}-\frac{m}{2}\right) & 0 & 2 \frac{z^{M}}{z^{4}} \rho^{M}\left(\partial_{s} x-m \frac{x}{2}\right) & \mathrm{i} \partial_{t}-A^{T} \\
0 & \mathrm{i} \frac{z^{M}}{z^{3}} \rho_{M}^{\dagger}\left(\partial_{s}-\frac{m}{2}\right) & \mathrm{i} \partial_{t}+A & -2 \frac{z^{M}}{z^{4}} \rho_{M}^{\dagger}\left(\partial_{s} x^{*}-m \frac{x}{2}\right)
\end{array}\right)
$$


where

$$
\begin{aligned}
A & =-\frac{6}{z} \phi+\frac{1}{z} \tilde{\phi}+\frac{1}{z^{3}} \rho_{N}^{*} \tilde{\phi}^{T} \rho^{L} z^{N} z^{L}+\mathrm{i} \frac{z^{N}}{z^{2}} \rho^{M N} \partial_{t} z^{M}, \\
\tilde{\phi} & \equiv\left(\tilde{\phi}_{i j}\right) \equiv\left(\phi_{j}^{i}\right) .
\end{aligned}
$$

In the simpler cases of Refs. $[9,10]$, a suitable choice of Yukawa terms turns out to ensure a definite positive Pfaffian, in connection with the relevant operator being real and antisymmetric and a double degeneracy observed in the spectrum, see comments in the next section. Here, the operator $O_{F}$ above is antisymmetric, and satisfies the constraint (reminiscent of the $\gamma_{5}$-hermiticity in lattice QCD) $[3,4]$

$$
O_{F}^{\dagger}=\Gamma_{5} O_{F} \Gamma_{5}
$$

where $\Gamma_{5}$ is the following unitary, antihermitian matrix

$$
\Gamma_{5}=\left(\begin{array}{cccc}
0 & \mathbb{1} & 0 & 0 \\
-\mathbb{1} & 0 & 0 & 0 \\
0 & 0 & 0 & \mathbb{1} \\
0 & 0 & -\mathbb{1} & 0
\end{array}\right), \quad \Gamma_{5}^{\dagger} \Gamma_{5}=\mathbb{1} \quad \Gamma_{5}^{\dagger}=-\Gamma_{5},
$$

which ensures that $\operatorname{det} O_{F}$ is real and non-negative. While the absence of a complex phase allows us to eliminate a systematic error of our previous analysis [1], it is not enough to make the Pfaffian definite positive, implying that the model may still suffer a sign problem - we comment on this in the next section.

\section{The fermionic spectrum}

As mentioned above, in simpler cases of models with four-fermion interactions $[9,10]$ a choice of Yukawa terms similar in spirit to the one described in the previous section turns out to ensure a definite positive Pfaffian. There the relevant operator is real and antisymmetric - so that its purely imaginary eigenvalues come in pairs $(i a,-i a)$ - and the key point there is that eigenvalues are also doubly degenerate. One may define the Pfaffian as the product of eigenvalues with positive imaginary part on the initial configuration. As the simulation progresses, sign flips in the Pfaffian correspond to an odd number of eigenvalues crossing through the origin, but as all eigenvalues are doubly degenerate such sign changes cannot occur, something that has been checked numerically. In our case one can check that - in the case of generally complex eigenvalues $\lambda$ - the properties (2.15) ensure a spectrum characterized by quartets $\left(\lambda,-\lambda^{*},-\lambda, \lambda^{*}\right)$, which would give a definite positive Pfaffian. However, for purely imaginary or purely real eigenvalues, the disposition in quartets is no longer enforced by (2.15) and indeed may not happen, leaving a spectrum of pairs $(\lambda,-\lambda)$ with no degeneracy. A numerical study of the spectrum of $O_{F}$ shows that the disposition in quartets would always occur only if the $A$-terms in (2.12) - defining Yukawa-like terms - were vanishing, see Figure 1 left, while for $A \neq 0$ (on the right) purely imaginary eigenvalues appear, with no degeneracy. One should notice that such purely imaginary eigenvalues appear also when auxiliary fields are set to zero - and thus the only non-vanishing $A$-term is the one present in the original Lagrangian, before linearization - suggesting that the sign ambiguity cannot be tamed by a suitably-enough choice of auxiliary fields. Figure 2, left panel, shows that the sign problem becomes severe for values of the coupling $g \sim 2$. Interestingly, the presence (case $r=1$ ) of a Wilson-like discretization [4] appears to shift its occurrence to lower $g$ [1]. One convenient way to answer the question of which region of the parameter space is free from a sign problem - and whether in such region information on the non-perturbative behavior of the system is obtainable - is to study the lowest part of the eigenvalue distribution for the fermionic operator and identify a region of the parameter space where zero eigenvalues of the determinant cannot occur. This is done in the right panel of Figure 2, which shows that the smallest eigenvalues are clearly separated 

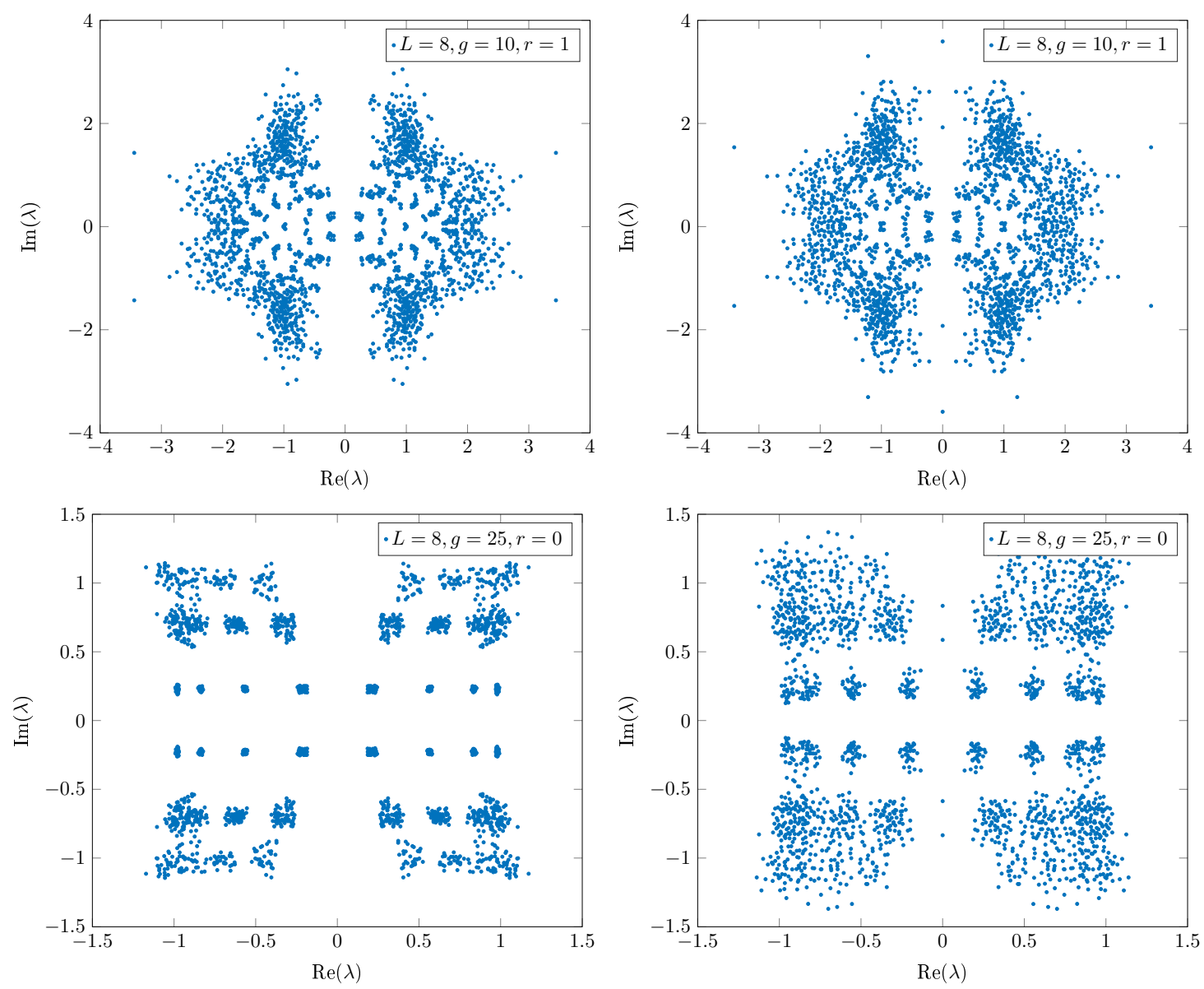

Figure 1: Spectrum of $O_{F}$, in absence (left diagrams) and presence (right diagrams) of $A$ (Yukawalike) terms in (2.12).

from zero for values of $g \geq 10$, where therefore sign flips cannot occur. It is interesting that this region safely includes $g=10$, at which (previous [4] and) current [1] simulations appear to detect the non-perturbative behavior, for example, of the (derivative of the) cusp anomaly as measured from the cusp action.

\section{Acknowledgments}

We are grateful to S. Catterall, R. Roiban, D. Schaich, K. Skenderis, R. Sommer and A. Wipf for very useful discussions.

\section{References}

[1] L. Bianchi, V. Forini, B. Leder, P. Töpfer, E. Vescovi, to appear.

[2] R. W. McKeown and R. Roiban, arXiv:1308.4875 [hep-th].

[3] V. Forini, L. Bianchi, M. S. Bianchi, B. Leder and E. Vescovi, "Lattice and string worldsheet in AdS/CFT: a numerical study,” PoS LATTICE 2015, 244 (2016) [arXiv:1601.04670 [hep-lat]].

[4] L. Bianchi, M. S. Bianchi, V. Forini, B. Leder and E. Vescovi, "Green-Schwarz superstring on the lattice,” JHEP 1607, 014 (2016) doi:10.1007/JHEP07(2016)014 [arXiv:1605.01726 [hep-th]]. 

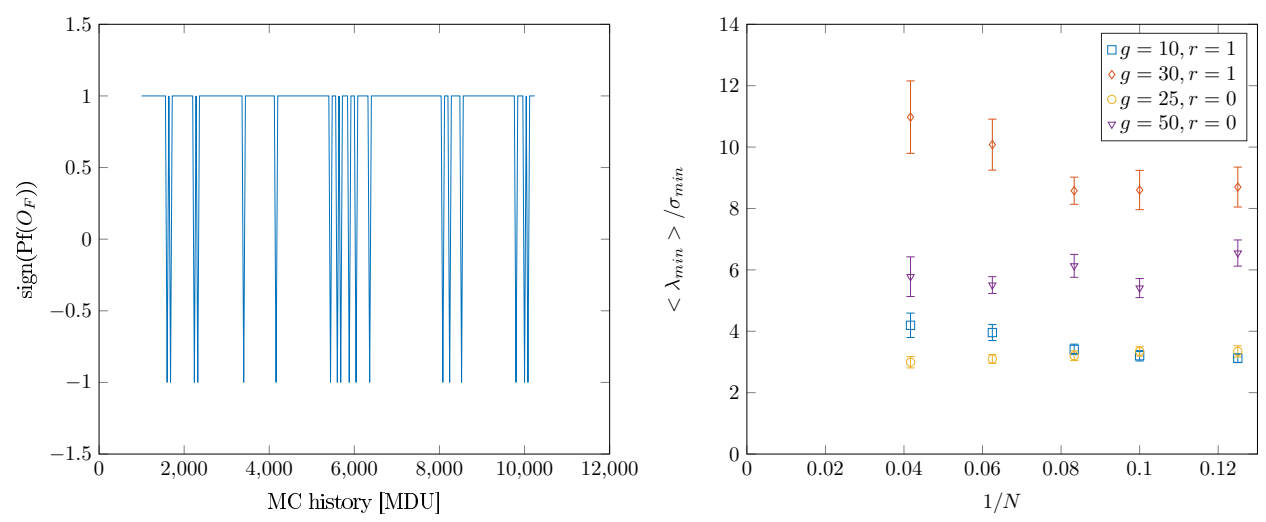

Figure 2: Left panel: Study of the sign of $\operatorname{Pf}\left(O_{F}\right)$ for lattice size $L=8$ and $g=2$. Right panel: The lowest part of the eigenvalue distribution $\lambda_{\min }$ of the quadratic (squared) fermion operator $O_{F}^{\dagger} O_{F}$ is well separated from zero. In the region of parameters explored, no zero eigenvalues for $\operatorname{det} O_{F}$ occur, which implies no sign flips, and therefore absence of sign problem, for its real Pfaffian.

[5] S. Catterall, D. B. Kaplan and M. Unsal, "Exact lattice supersymmetry," Phys. Rept. 484, 71 (2009) • D. Schaich, "Aspects of lattice $\mathscr{N}=4$ supersymmetric Yang-Mills", PoS LATTICE 2015242 (2015) arXiv:1512.01137 [hep-lat] • G. Bergner and S. Catterall, "Supersymmetry on the lattice," Int. J. Mod. Phys. A 31, no. 22, 1643005 (2016) [arXiv:1603.04478 [hep-lat]]. • D. Schaich, S. Catterall, P. H. Damgaard and J. Giedt, "Latest results from lattice N=4 supersymmetric Yang-Mills," PoS LATTICE 2016, 221 (2016) [arXiv:1611.06561 [hep-lat]]. • E. Berkowitz, E. Rinaldi, M. Hanada, G. Ishiki, S. Shimasaki and P. Vranas, Phys. Rev. D 94, no. 9, 094501 (2016) doi:10.1103/PhysRevD.94.094501 [arXiv:1606.04951 [hep-lat]].

[6] R. R. Metsaev and A. A. Tseytlin, "Superstring action in $A d S_{5} \times S^{5}$. Kappa symmetry light cone gauge," Phys. Rev. D 63, 046002 (2001) ; R. R. Metsaev, C. B. Thorn and A. A. Tseytlin, Nucl. Phys. B 596, $151(2001)$

[7] S. Giombi, R. Ricci, R. Roiban, A. A. Tseytlin and C. Vergu, "Quantum AdS(5) x S5 superstring in the AdS light-cone gauge," JHEP 1003, 003 (2010)

[8] N. Beisert, B. Eden and M. Staudacher, "Transcendentality and Crossing," J. Stat. Mech. 0701, P01021 (2007) • B. Basso, "Exciting the GKP string at any coupling," Nucl. Phys. B 857, 254 (2012) [arXiv:1010.5237 [hep-th]].

[9] S. Catterall, "Fermion mass without symmetry breaking," JHEP 1601, 121 (2016) doi:10.1007/JHEP01(2016)121 [arXiv:1510.04153 [hep-lat]].

[10] S. Catterall and D. Schaich, "Novel phases in strongly coupled four-fermion theories," arXiv:1609.08541 [hep-lat]. 